\title{
Energy Saving Mechanisms in Sensor Networks
}

\author{
Lan Wang and Yang Xiao \\ Computer Science Department \\ The University of Memphis \\ 373 Dunn Hall \\ Memphis, TN 38152 USA \\ Emails: lanwang@memphis.edu, yangxiao@ieee.org
}

\begin{abstract}
In this paper, we provide a survey on energyefficient scheduling mechanisms in sensor networks that have different design requirements than those in traditional wireless networks. We classify these mechanisms based on their design assumptions and design objectives. Different mechanisms may make different assumptions about their sensors including detection model, sensing area, transmission range, failure model, time synchronization, and the ability to obtain location and distance information. They may also have different assumptions about network structure and sensor deployment strategy. Furthermore, while all the mechanisms have a common design objective to maximize network lifetime, they may also have different objectives determined by their target applications.
\end{abstract}

Keywords: Sensor Network, Energy Consumption, Sleep-mode, Scheduling

\section{INTRODUCTION}

Sensor networks have a wide variety of applications in both military and civil environment [1]. Some of these applications, e.g., natural habitat monitoring, require a large number of tiny sensors and these sensors usually operate on limited battery power. According to [8], individual sensors can last only 100120 hours on a pair of AAA batteries in the active mode. On the other hand, since the number of sensors is huge and they may be deployed in remote, unattended, and hostile environments, it is usually difficult, if not impossible, to recharge or replace their batteries. This problem is compounded by the fact that battery capacity only doubles in 35 years [19].

Since a sensor network is usually expected to last several months to one year without recharging [18], [25], optimal energy consumption, i.e., minimizing energy consumed by sensing and communication to extend the network lifetime, is an important design objective. In the meantime, how well a sensor network can collect sensory data depends on its sensing coverage and network connectivity. Therefore, maintaining sufficient sensing coverage and network connectivity are important design requirements for sensor networks. Furthermore, fault tolerance should also be considered when minimizing energy consumption in the presence of individual sensor failure [16]. In fact, there are many design objectives for a sensor network, some of which are summarized in Section IIB.

To minimize energy consumption and extend network lifetime, a common technique is to put some sensors in the sleep mode and put the others in the active mode for the sensing and communication tasks. When a sensor is in the sleep mode, it is shut down except that a low-power timer is on to wake up the sensor at a later time [11], therefore it consumes only a tiny fraction of the energy consumed in the active mode ([8], [18]). Moreover, in cluster-based networks, cluster heads are usually selected in a way that minimizes the total energy consumption and they may rotate among the sensors to balance energy consumption.

Both approaches try to save energy by configuring the sensors into certain topologies, therefore such mechanisms have been referred to as "topology configuration" mechanisms. In this paper, we call them "energy-efficient scheduling mechanisms". There are many other methods to save energy, such as reducing communication range and reducing control messages, but we only discuss distributed scheduling mechanisms in this paper.

Although there are many scheduling mechanisms published in the literature, different mechanisms often have different assumptions, mainly because they are considered in the context of different applications. The design assumptions include, but are not limited to, detection model, sensing area, transmission range, failure model, time synchronization, location information, and distance information. There are also different assumptions about network structure and sensor deployment strategy. Furthermore, while all the mechanisms have a common design objective to maximize network lifetime, they may also have different objectives determined by their target applications. For example, a surveillance application may require the working sensors to achieve a certain degree of sensing coverage. Other design objectives include network connectivity, high data delivery ratio, high quality of surveillance, stealthiness, balanced energy consumption, scalability, robustness, and simplicity.

It is unfair and sometimes misleading to compare the scheduling mechanisms without considering the different assumptions and objectives, yet they are often compared without taking into consideration these factors. Therefore, we believe that the first step to understand the differences among the mechanisms is to understand their assumptions and objectives. To this end, we have surveyed 15 distributed scheduling mechanisms designed for sensor networks and present the results in this paper. This is by no means a complete survey of the literature. Rather, we hope that this set of sample mechanisms could help us understand the wide range of design choices. 
The rest of the paper is organized as follows. Section 2 discusses the design assumptions and objectives. Section 3 explains the different energy consumption modes that a sensor can use. Section 4 and Section 5 review the scheduling mechanisms in non-hierarchical networks and hierarchical networks, respectively. After understanding all the schemes, we provide a classification in Section 6. We conclude this survey in Section 7 .

\section{Classification Methodology}

All the surveyed mechanisms have a common objective - maximizing sensor network lifetime. However, they make quite different assumptions regarding the sensors and the sensor network. They also have different objectives that are determined by their applications. Therefore, a fair comparison among the surveyed mechanisms has to take into consideration these factors. In this section, we summarize the design assumptions and objectives. Based on this information, any classification can be easily derived. We will present the result of our classification in Section 6 after reviewing all the mechanisms.

\section{A. Design Assumptions}

Since the focus of this survey is energy saving, all the surveyed mechanisms make the following common assumptions: (1) each sensor has limited energy supply and (2) the sensor network is expected to run for a long time. Below we discuss the different design assumptions that reflect different network structures, sensor deployment strategies, and sensor capabilities (see Figure 1).

a) Network Structure: A sensor network can be nonhierarchical or flat in the sense that every sensor has the same role and functionality. Alternatively, a sensor network can be hierarchical. For example, in sensor networks designed for detection and tracking, some sensors may be designated as the fusion centers: they collect the reports from the sensors in their neighborhood, make a decision regarding whether an object has been detected, and send a report to the base station. These networks are often cluster-based (or sentry-based) in which the cluster-heads (or sentries) have a more prominent role than the other sensors. Several surveyed mechanisms, e.g., [11], assume that the sensor network is cluster-based. The detailed survey in Section 4 and Section 5 is in fact organized (at the top level) based on whether the mechanism makes this assumption or not.

b) Sensor Deployment Strategy: The performance of a sensor network, e.g., its sensing coverage, can be affected by how the sensors are initially placed. There are various sensor placement mechanisms. For example, sensors may be dispersed from an airplane flying over the sensing field, and they may also be manually placed at selected locations. In the first scenario, the sensors' locations are likely to follow a random distribution. Several surveyed mechanisms [25], [27] assume that the sensors are randomly and uniformly distributed over the sensing field. Sometimes a mechanism does not explicitly state this assumption, but its performance is best when this assumption holds. Some papers also use the two-dimensional Poisson distribution [18]. In addition, a few mechanisms assume that the sensors form a grid [18], [21].

Most of the surveyed mechanisms assume that there are some redundant sensors in the network that can be turned off. One explicitly assumes that the total number of sensors is orders of magnitude higher than the number of working sensors [27]. We consider this level of density to be "high". Otherwise, if the two numbers are on the same order, we consider the level of density to be "normal".

c) Detection Model: Most surveyed mechanisms assume that a sensor can detect an object as long as the object is inside its sensing range, i.e., the detection model is deterministic. However, one notable exception is [26] - it uses a probabilistic detection model in which the detection probability of an object is a function of the distance between the object and the sensor.

d) Sensing Area: The sensing area is usually assumed to be either a circular area or a 3-D sphere. Moreover, the sensors are usually assumed to have the same sensing range. There are several mechanisms that are extensible to any convex and non-uniform (but still deterministic) sensing areas [4].

e) Transmission Range: Several mechanisms [11], [27] assume that a sensor's radio transceiver is capable of changing its transmission power in continuous steps to achieve different transmission ranges. Some sensors, such as the MICA2 mote [7], provide multiple levels of transmission power.

f) Time Synchronization: Several mechanisms assume that sensors are time synchronized so that they can wake up at the same time to start a new round of scheduling [18], [22], [28]. Many time synchronization algorithms have been proposed for sensor networks (see [12] for an example).

g) Failure Model: How nodes may fail is an important assumption about both the sensors and the environment in which they are deployed. All the surveyed mechanisms assume that a sensor fails when its energy is depleted. Several mechanisms, e.g., [27], further assume that sensors may fail unexpectedly before they run out of battery. For example, the sensors may be destroyed by tanks if they are spread in a military field.

h) Sensor Mobility: All the surveyed mechanisms either assume explicitly that the sensor network is stationary or do not make an explicit assumption about mobility. In fact, several papers, e.g., [25], argue that most real-world sensor networks involve little or no mobility.

i) Location Information: Several surveyed mechanisms assume that sensors can identify their geographic locations. The location information is usually used to determine whether (and how much) a node's sensing area overlaps with its neighbors' sensing areas. If the location for each sensor is predetermined and the sensors are not mobile, then the location information can be hard-coded in the sensors before they are deployed. Otherwise, sensors may need to be equipped with GPS devices or run a localization algorithm such as the one proposed in [5].

j) Distance Information: [9] and [11] assume that sensors in a cluster-based sensor network can determine the 


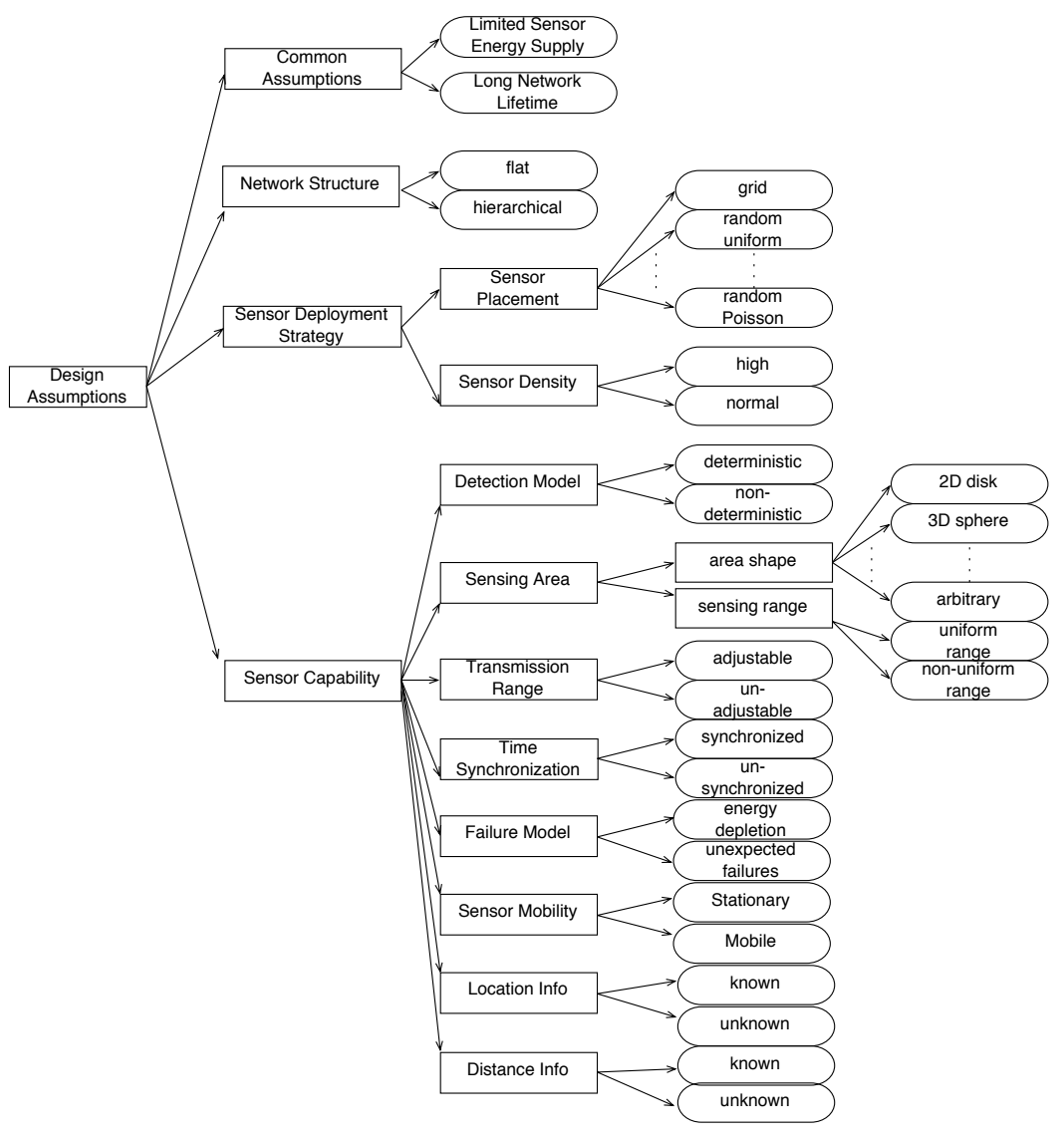

Fig. 1. Design Assumptions of Scheduling Mechanisms

distance to their cluster head. Distance information can be obtained via location information (but the reverse is not true). In addition, distance information may be inferred from the strength of received signals [11].

\section{B. Design Objectives}

Applications differ in their requirements, therefore the underlying sensor networks usually have different design objectives or have different priorities among the objectives. Maximizing network lifetime is certainly one of the most important design objectives for all the sensor networks that need to run for a long time. A sensor network, however, is built to accomplish certain tasks, e.g., to perform sensing and deliver sensory data. Therefore, one or more Quality of Service objectives, such as maintaining sensing coverage, are usually considered along with minimizing energy consumption. Furthermore, a design may consider high-level objectives such as robustness, scalability and simplicity. Since a design decision for achieving one objective may have an impact on some of the other objectives, we not only summarize the objectives in this section, but also discuss the relationship among them.

a) Maximizing Network Lifetime: Network lifetime has been defined in various ways [6], [11], [25], [27], [14] and an energy-efficient mechanism may choose to maximize a certain type of network lifetime. In the simplest case, a network may be considered alive when any of the sensors is alive. Network lifetime can also be calculated as the duration of time when the percentage of sensors that have depleted their energy is below a threshold, e.g., 90\% [6], [11]. Alternatively, one or more quality of service measures can be taken into account. For example, a network may be considered functional only when its sensing coverage (or degree of connectivity, data delivery ratio, etc.) is above a certain threshold [25], [27].

b) Sensing Coverage: Since sensing is the primary function of a sensor network, sensing coverage is an important QoS measure of the network. If every point in the field is monitored by at least 1 sensor, the sensor network is said to achieve 1-coverage. If every point in the field is monitored by at least $K$ sensors, the sensor network is said to achieve $K$ coverage [27] (1-coverage is a special case of $K$-coverage). A sensor network may also provide partial 1-coverage or partial $K$-coverage. Sometimes a deterministic guarantee may not be possible under the chosen assumptions. Therefore, a few mechanisms ensure asymptotic coverage when the number of sensors goes to infinity [18].

c) Network Connectivity: If sensory data need to travel multiple hops to reach the destination (e.g., the base station), it is important to maintain the connectivity among the sensors. Some mechanisms, e.g., [24], can even configure the network to a specific degree of connectivity required by the application, 


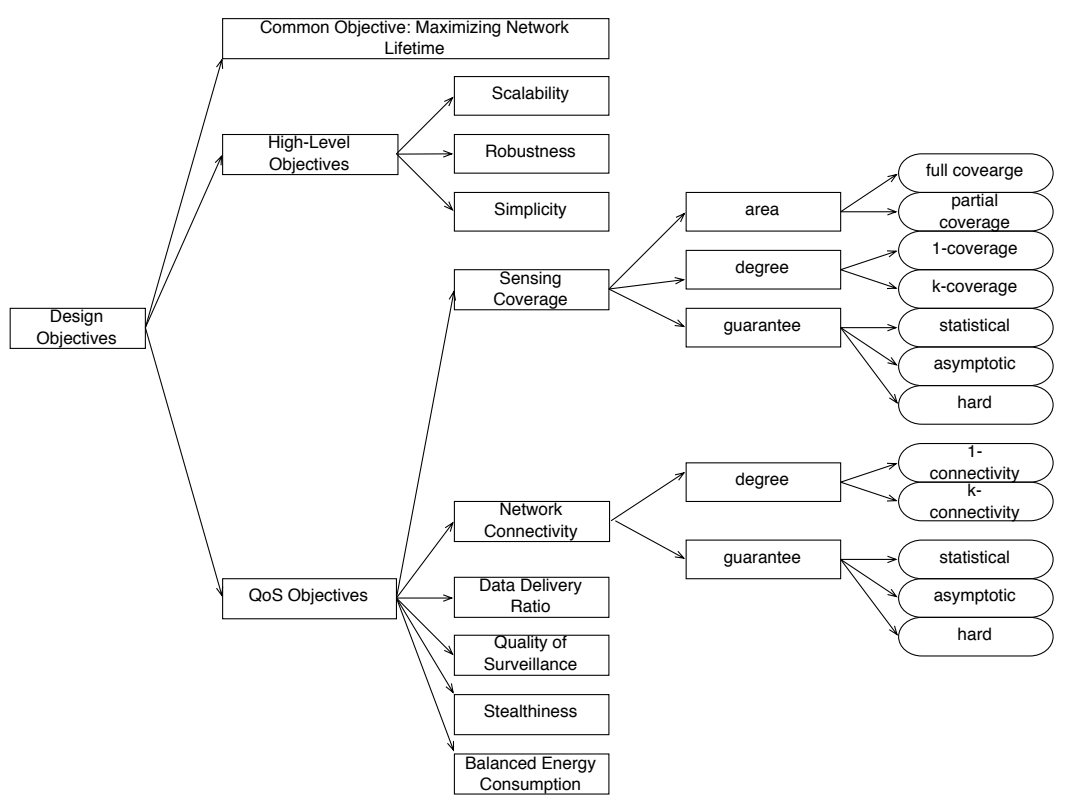

Fig. 2. Design Objectives of Scheduling Mechanisms

given a sufficiently high sensor density. Similar to sensing coverage, connectivity can also be achieved in an asymptotic sense, i.e., the network is guaranteed to be connected when there is an infinite number of sensors [27].

d) Data Delivery Ratio: A high data delivery ratio is another desirable QoS objective for some applications. It can be measured by the average percentage of data delivered from a source to a sink. However, this measure is not appropriate when there is in-network data aggregation.

e) Quality of Surveillance: Gui and Mohapatra proposed a metric, Quality of Surveillance (QoSv), to measure the performance of target-tracking sensor networks [13]. QoSv is defined as the inverse of the average distance traveled by a target before it is detected by a sensor. This definition implies that if a sensor network can detect a moving target within a shorter distance following the target's intrusion than other networks, it is considered to have a higher quality of surveillance. QoSv is not purely a function of sensing coverage. It also depends on other factors such as the sensors' geographical distribution. If the sensors are clustered in certain areas, then targets entering the network from the other areas can travel a long distance before they are detected. Therefore, the average distance traveled by a random target before its detection in this network may be longer than in another network that has the same sensing coverage but whose sensors are more evenly distributed over its entire area.

f) Stealthiness: Certain surveillance applications require the operations of the sensor network to be stealthy or less likely to be detected by others. Stealthiness can be achieved by reducing the number of control messages. Shortening the communication period may also improve stealthiness, if the sensors communicate with each other only during that period [14]. g) Balanced Energy Consumption: Some mechanisms strive to balance the energy consumption among the sensors. One common argument for doing this is that if the energy of certain nodes is depleted before the others, holes may appear in the sensing coverage or the sensor network may become disconnected prematurely. A counter argument is based on the assumption of high sensor density (see Section II-A for the design assumptions): even if those nodes die prematurely, there will still be some redundant nodes that can be turned on at or near those locations.

h) Scalability: There is no universal definition of scalability. However, it is generally undesirable for sensors to have a state overhead or computation overhead that increases linearly or even faster with the number of all potential neighbors. On the other hand, if each node needs to keep track of only active neighbors and the number of active neighbors is small at any given time, the mechanism may still be considered scalable.

i) Robustness: Robustness is the ability of a network to withstand unexpected failures. For example, sensor nodes on a battle field may be destroyed by tanks and bombs before their batteries run out. A robust mechanism cannot expect everything to go as planned. For an instance, it cannot expect all the sleeping nodes to wake up - some of these nodes may have stopped functioning while they were sleeping. Obviously, the assumptions made by the designers regarding possible failures can have a significant impact on the robustness of a mechanism.

j) Simplicity: Current sensors have very limited memory space for storing programs, e.g., the MICA2 mote has only $8 \mathrm{~KB}$ of memory for this purpose. Moreover, they usually have limited computation power and they are difficult to debug. Therefore, simpler mechanisms are more likely to be deployed in sensor networks. 
k) Relationship between Design Objectives: Since it is impossible to enumerate all the possible relationships among the design objectives, we list some of the important ones here: (1) when sensors' transmission range is at least twice their sensing range, ensuring $K$-coverage can lead to $K$ connectivity [24], [28]. However, the reverse is not necessarily true; (2) a higher degree of connectivity generally leads to a higher degree of robustness against failures, since more links need to be removed to make the network disconnected; (3) data delivery ratio normally improves as the degree of connectivity increases. However, if the connectivity degree is too high, the data collisions among sensors may adversely affect the delivery ratio; (4) a higher degree of stealthiness may indicate lower energy consumption and improved data delivery ratio, if stealthiness is achieved through minimizing control messages; (5) a simpler mechanism tends to be more robust because the programmer is less likely to make a mistake.

\section{ENERgy SAVING Modes of SEnsors}

In order to understand the surveyed mechanisms, one first needs to be able to differentiate the various energy saving modes that can be provided by a sensor. One complexity here is that different types of sensors may support different sets of modes and even if they support the same set of modes, they often use different terminology. To make our presentation clear, we define the major modes of a sensor as follows:

- on-duty: all the components in the sensor are turned on. The sensor is able to collect sensory data, send/receive messages, process data and messages, and do other types of computation. This mode is also called the active mode in the literature. It is not an energy-saving mode.

- sensing unit on-duty: at least one sensing unit and the processor are turned on, but the transceiver is turned off. In this mode, the sensor is capable of sensing and processing sensory data, but not transmitting or receiving messages. We also use the shorter form SU-on-duty in the paper.

- transceiver on-duty: the transceiver and the processor are turned on, but all the sensing units are turned off. In this mode, the sensor is capable of transmitting, receiving and processing messages, but not sensing. We also use the shorter form TR-on-duty in the paper.

- off-duty: the sensor's processor is turned off, but a timer or some other triggering mechanism may be running to wake up the sensor. This mode is also called the sleep mode in the literature.

Note that some sensors have multiple off-duty (sleep) modes, each with a different wakeup mechanism. For example, the $\mu$ AMPS sensor has three sleep modes: Monitor, Observe, and Deep Sleep [20]. The processor is turned off in all three modes, so the sensor cannot process any sensory data or messages. However, in the Monitor mode, both the sensing unit and the transceiver are left on to receive wakeup signals. In the Observe mode, only the sensing unit is on. Note that the Observe mode is different from the SU-on-duty mode as the processor is turned on in the latter. In the Deep Sleep mode, neither the sensing unit nor the transceiver is turned on, so the sensor relies on a preset internal timer to wake itself up. Most sensors provide a sleep mode similar to $\mu$ AMPS' Deep Sleep mode and this sleep mode is exploited by most energy-saving mechanisms. However, the designers of $\mu$ AMPS proposed a mechanism to take advantage of all of its three sleeping modes [20].

Sensors save the most energy in the (Deep Sleep) offduty mode since the computing, sensing and communication components are turned off. They generally save more energy in the SU-on-duty mode than in the TR-on-duty mode, because communication usually consumes more energy than sensing [7], [17]. However, the energy consumption in the TR-on-duty mode also depends on the actual communication pattern and frequency.

Almost all of the surveyed mechanisms take advantage of the energy saving feature of the off-duty mode. Some mechanisms also exploit the SU-on-duty and TR-on-duty modes. Note that sometimes it is not easy to identify which energy-saving mode that a mechanism uses. For example, in [4], sensors cannot be completely turned off, but they can enter an idle mode in which they are only required to listen to messages. However it is not clear whether this idle mode refers to the Monitor mode of $\mu$ AMPS (an off-duty mode) or the TR-on-duty mode.

\section{Distributed Scheduling Mechanisms in NON-HIERARCHICAL NETWORKS}

In this section, we briefly review nine scheduling mechanisms that can be applied to non-hierarchical sensor networks. Readers can find more details in the extended version of this survey [23].

In [18], Kumar et al. adopt the Randomized Independent Scheduling (RIS) mechanism to extend network lifetime while achieving asymptotic $K$-coverage. RIS assumes that time is divided into cycles based on a time synchronization method. At the beginning of a cycle, each sensor independently decides whether to become active with probability $p$ or go to sleep with probability $1-p$. Thus the network lifetime is increased by a factor close to $\frac{1}{p}$ (i.e., $p$ determines the network lifetime). Furthermore, Kumar et al. derived the conditions for asymptotic $K$-coverage when RIS is used with three different sensor deployment strategies - grid, random uniform, and 2dimensional Poisson.

Tian and Georgana proposed a distributed scheduling mechanism to save energy while preserving sensing coverage [22]. To avoid reducing sensing coverage, this mechanism allows a sensor to turn off only if its sensing area is completely covered by its neighbors' sensing areas - the neighbors are called this node's off-duty sponsors in this case and the sector that a neighbor covers in its sensing area is called a sponsored sector. Each sensor uses its neighbors' location information and sensing range to determine the sponsored sectors and concatenate the central angles of the sponsored sectors. If the entire 360 degrees of the central angle are covered, then the 
node is eligible to enter the off-duty mode. We refer to this mechanism as Sponsored Sector in the rest of this paper.

Berman et al. [4] formulated the sleep-scheduling problem as a maximization problem with constraints on battery lifetime and sensing coverage. They also presented a centralized and a distributed algorithm to maximize network lifetime while achieving $K$-coverage. Their distributed mechanism can guarantee a specific degree of sensing coverage (assuming that the sensor density is high enough) whereas the aforementioned Sponsored Sector mechanism preserves the existing coverage degree. In this mechanism, each sensor is in one of three states: active, idle or vulnerable. In the vulnerable state, if the sensor discovers that part of its sensing area cannot be covered by any of its active or vulnerable neighbors, it immediately enters the active state. Otherwise, it enters the idle state if its sensing area can be monitored by either active neighbors or vulnerable neighbors with a higher energy level.

In [25], Wu et al. proposed a distributed scheduling mechanism called LDAS (Lightweight Deployment-Aware Scheduling). Unlike previous studies, this work assumes that sensor nodes are not equipped with GPS or other devices to obtain location information. Since it is difficult, if not impossible, to determine whether a node's sensing area is absolutely covered by other nodes without location information, the goal here is to provide statistical guarantees on sensing coverage. Note that [27] does not assume the knowledge of location information either, but this work is complementary to [27] as the latter guarantees asymptotic network connectivity. LDAS assumes that each working node has a mechanism to know the number of working nodes in its neighborhood. When the number of working neighbors exceeds a threshold determined by the application's requirement on sensing coverage, the node randomly selects some of its neighbors to turn off and sends tickets to them. When a node collects enough tickets from its neighbors, it may enter the off-duty mode after a random back-off period.

Ye et al. developed a mechanism called PEAS (Probing Environment and Adaptive Sensing) that can extend the lifetime of a high-density sensor network in a harsh environment [27]. What distinguishes this work from the previous studies are first its assumptions. First, it assumes that sensor nodes may fail frequently and unexpectedly, which makes synchronized sleeping algorithms infeasible because they depend on the predictability of sensors' lifetime. Second, it assumes that the sensor network is so dense that the total number of sensors may be orders of magnitude higher than the number of working nodes. As a result, it is infeasible for nodes to maintain per-neighbor state. Finally, it assumes that nodes do not have location information. The authors argue that these assumptions lead to a design that is more robust against failures and easier to implement in a real sensor network. PEAS conserves energy by separating all the working nodes by a minimum distance of $c$. To check if there is a working neighbor nearby, each node broadcasts a message (probe) with a transmission range of $c$ after sleeping for a random period. A node will enter the on-duty mode only if it receives no replies from working neighbors; otherwise it will stay in the off-duty mode. In the same paper, Ye et. al. proved that PEAS can guarantee asymptotic connectivity by satisfying two conditions on the sensor density and the probing range.

Zhang and Hou proved that 1-coverage implies 1connectivity when the ratio between the radio transmission range and the sensing range is at least two [28]. Assuming that this condition is satisfied, Zhang and Hou further proposed a distributed mechanism, Optimal Geographic Density Control (OGDC), to maximize the number of sleeping sensors while ensuring that the working sensors provide complete 1-coverage and 1-connectivity[28]. OGDC tries to minimize the overlapping area between the working sensors. A sensor is turned on only if it minimizes the overlapping area with the existing working sensors and if it covers an intersection point of two working sensors. A sensor can verify whether it satisfies these conditions using its own location and the working sensors' locations. OGDC is quite similar to the Sponsored Sector mechanism, except that they use different on-duty/off-duty eligibility rules and the Sponsored Sector mechanism is more conservative when turning off sensors.

Wang et al. proposed an integrated coverage and connectivity configuration protocol called CCP [24]. This protocol aims to maximize the number of sleeping nodes, while maintaining both $K$-coverage and $K$-connectivity. Note that the OGDC mechanism ensures 1-coverage and 1-connectivity. CCP's capability is based on the theorem that $K$-coverage implies $K$ connectivity when the transmission range is at two times the sensing range (this theorem is proved in [24]). To ensure $K$ coverage, a node only needs to check whether the intersection points inside its sensing area are $K$-covered (based on a theorem proved in [24]).

In [6], Cerpa and Estrin proposed using sensors' local measurements to automatically configure network topology in a high density sensor network. The goal is to maintain a certain data delivery ratio while allowing redundant sensors to stay asleep in order to conserve energy. Achieving this goal requires configuring the network to the right level of connectivity; it cannot be too low to hamper data delivery, but it cannot be too high either since neighboring nodes might interfere with each other leading to a high collision rate. The approach adopted by ASCENT is to let sensors measure their connectivity as well as their data loss rate and activate their neighbors based on these local measurements.

PECAS is proposed by Gui and Mohapatra in [13]. It is an extension of the PEAS protocol proposed in [27]. It has the same environment probing mechanism as PEAS, but it does not let working nodes stay awake indefinitely. The designers argue that "the failure of nodes (due to energy depletion) may cause partitioning of the network or isolation of nodes ... it is desirable to balance energy consumption among the neighboring nodes." Therefore, a working node in PECAS will go back to sleep after a specified period of time. It also advertises the remaining working time in its reply messages to its neighbors' probe messages. In this way, a working neighbor who decides to enter the off-duty mode can schedule itself to 
wake up before this node goes to sleep, thus preventing the occurrence of blind spots.

\section{Distributed Scheduling Mechanisms in HIERARCHICAL NETWORKS}

In a hierarchical network such as a cluster-based network, sensors organize themselves into local clusters by some mechanisms. Each cluster has a cluster head. The cluster head may or may not be a more powerful sensor/device than other sensors. The cluster head manages the sensors in its own cluster for communications between the cluster and the base station. Communication between cluster heads and the base station may be multi-hop through other cluster heads. In this section, we briefly introduce seven cluster-based scheduling mechanisms as follows. Readers are referred to an extended version of this survey [23] for more information.

Heinzelman et al. [16] proposed Low-Energy Adaptive Clustering Hierarchy (LEACH), a clustering-based protocol utilizing randomized rotation of cluster heads to evenly distribute the energy load among the sensors. In LEACH, the operation is divided into cycles. Since cluster heads have more responsibilities and consume more energy, LEACH let different sensors become cluster heads in each cycle to prevent the cluster heads from running out of energy first. Cluster heads are self-elected at the beginning of each cycle. To conserve energy, non-head sensors are turned off at all times except during their transmission time.

The journal version of LEACH [15] further improved LEACH in two major aspects: (1) it proposed better cluster head selection algorithms; and (2) it determined the optimal number of clusters. We refer to this mechanism as Enhanced Low-Energy Adaptive Clustering Hierarchy or E-LEACH.

Bandyopadhyay et al. [2], [3] considered a simple strategy to select cluster heads - they are chosen randomly with a probability $p$. There are two kinds of cluster heads: volunteer cluster heads and forced cluster heads. Each sensor can become a volunteer cluster head with probability $p$. A volunteer cluster head advertises itself to the neighboring sensors, which then forward the advertisement within $k$ hops. Any noncluster-head sensor that receives such advertisements joins the cluster of the closest cluster head. Any sensor not associated with a cluster within $t$ units of time becomes a forced cluster head. Bandyopadhyay et al. [2], [3] further determined the optimum value of $p$ to minimize energy consumption in an $h$-level hierarchical sensor network.

$\mathrm{He}$ et al. [14] designed the Energy-Efficient Surveillance System (ESS), in which the trade off between energy consumption and surveillance performance is explored by adaptively adjusting the sensitivity of the system. In [14], sensors are classified into sentries and non-sentries dynamically over time. The sentries (similar to cluster heads) are elected locally by each sensor, using the information gathered from its neighbors. A sensor decides to become a sentry if it discovers that none of its neighbors is a sentry, and it advertises its intent. A random backoff delay is used to avoid collisions when multiple sensors in the same neighborhood advertise their intent at the same time. The backoff delay of a sensor is set inversely proportional to its residual energy for balanced energy consumption [14]. The non-sentry sensors alternate between sleep and wake-up states. Two different schemes, proactive control and reactive control, to determine the sleepwakeup cycle are described in [14].

Deng et al. [11] proposed a sleep-scheduling algorithm, called Linear Distance-based Scheduling (LDS) scheme for cluster-based high density sensor networks. The goal is to reduce energy consumption while maintaining adequate sensing coverage capabilities [11]. To achieve this goal, the LDS scheme selects sensors farther away from the cluster head to sleep with higher probabilities. The rationale behind this scheme is based on the assumption that each sensor's radio transceiver is capable of changing its transmission power in continuous steps to achieve different transmission ranges; a farther away sensor needs more power to communicate with the cluster head, and therefore, has higher energy consumption. The LDS scheme only considers static clusters. In other words, cluster heads are not changed once they are selected.

Deng et al. proposed Balanced-energy Sleep Scheduling (BS) in [10]. BS extends the LDS scheme by evenly distributing the sensing and communication tasks among the non-head sensors so that their energy consumption is similar regardless of their distance to the cluster-head. More specifically, the authors derived a sleep probability function $p(x)$ so that the total energy consumption of a sensor does not depend on $x$, the distance between the sensor and its cluster head.

\section{Classification}

Since there are many dimensions along which to classify the mechanisms, we do not attempt to enumerate all the possible combinations. Rather, we summarize the choices made by the surveyed mechanism and this summary can be used as an input to any classification system.

Extracting the design assumptions and objectives from the papers is not easy as we first expected. The designers usually state explicitly the most important assumptions and objectives, but we have to extrapolate the remaining ones and our extrapolation may not reflect the designers' original intentions accurately. To minimize this problem, we do not include in our summary the high-level design objectives, i.e., robustness, simplicity and scalability, which are more difficult to quantify.

Our results are presented in Table I and Table II. In table II, we consider a mechanism to achieve stealthiness if it satisfies one of the following conditions:

1) no communication is required for sleep scheduling. For example, in the RIS mechanism, each node decides whether to go to sleep using a pre-determined probability;

2) The operation is divided into rounds and communication among sensors to set up schedules is required only at the beginning of a round.

Moreover, a mechanism is considered to achieve balanced energy consumption if sensors rotate to become on-duty and off-duty. 
TABLE I

ClASSIFICATION BASED ON AsSUMPTIONS

\begin{tabular}{|c|c|c|c|c|c|c|c|c|c|c|c|c|}
\hline \multirow[b]{2}{*}{ Schemes } & \multicolumn{12}{|c|}{ Assumptions } \\
\hline & $\begin{array}{l}\text { Network } \\
\text { Structure }\end{array}$ & $\begin{array}{c}\text { Sensor } \\
\text { Placement }\end{array}$ & $\begin{array}{l}\text { Sensor } \\
\text { Density }\end{array}$ & $\begin{array}{l}\text { Determi- } \\
\text { nistic } \\
\text { Detection }\end{array}$ & $\begin{array}{c}\text { Sensing } \\
\text { Area }\end{array}$ & $\begin{array}{l}\text { Uniform } \\
\text { Sensing } \\
\text { Range }\end{array}$ & $\begin{array}{c}\text { Adjustable } \\
\text { Transmission } \\
\text { Range }\end{array}$ & $\begin{array}{l}\text { Time } \\
\text { Synch. }\end{array}$ & $\begin{array}{c}\text { Frequent } \\
\text { Failures }\end{array}$ & Mobility & $\begin{array}{c}\text { Known } \\
\text { Location }\end{array}$ & $\begin{array}{c}\text { Known } \\
\text { Distance }\end{array}$ \\
\hline $\begin{array}{c}\text { RIS } \\
\text { (Kumar) }\end{array}$ & Flat & $\begin{array}{c}\text { Grid, } \\
\text { Uniform, } \\
\text { Poisson }\end{array}$ & $\begin{array}{l}\text { Normal, } \\
\text { High }\end{array}$ & Yes & 2-D Disk & Yes & No & Yes & No & No & No & No \\
\hline $\begin{array}{l}\text { Sponsored } \\
\text { Sector }\end{array}$ & Flat & Any & $\begin{array}{c}\text { Normal, } \\
\text { High }\end{array}$ & Yes & 2-D Disk & Yes & No & Yes & No & No & $\overline{\text { Yes }}$ & No \\
\hline MSNL & Flat & Any & $\begin{array}{l}\text { Normal, } \\
\text { High }\end{array}$ & Yes & $\begin{array}{c}2-\mathrm{D} \\
\text { Convex }\end{array}$ & No & No & No & No & No & Yes & No \\
\hline LDAS & Flat & Uniform & $\begin{array}{l}\text { Normal, } \\
\text { High }\end{array}$ & $\overline{\text { Yes }}$ & 2-D Disk & Yes & No & No & No & No & No & No \\
\hline PEAS & Flat & Uniform & High & Yes & Any & Any & Yes & No & Yes & No & No & No \\
\hline OGDC & Flat & Any & $\begin{array}{c}\text { Normal, } \\
\text { High }\end{array}$ & Yes & 2-D Disk & Yes & No & Yes & No & No & Yes & No \\
\hline $\mathrm{CCP}$ & Flat & Any & $\begin{array}{c}\text { Normal, } \\
\text { High }\end{array}$ & Yes & 2-D Disk & Yes & No & No & No & No & Yes & No \\
\hline ASCENT & Flat & Any & High & Yes & Any & Any & No & No & No & No & No & No \\
\hline PECAS & Flat & Uniform & $\begin{array}{c}\text { Normal, } \\
\text { High }\end{array}$ & Yes & Any & Any & Yes & No & Yes & No & No & No \\
\hline LEACH & Hierarchical & Any & $\begin{array}{c}\text { Normal, } \\
\text { High }\end{array}$ & Yes & Any & Any & No & $\overline{\text { Yes }}$ & No & No & No & No \\
\hline E-LEACH & Hierarchical & Any & $\begin{array}{c}\text { Normal, } \\
\text { High }\end{array}$ & Yes & Any & Any & No & Yes & No & No & No & No \\
\hline $\begin{array}{l}\text { Bandyo- } \\
\text { padhyay et } \\
\text { al. [2], [3] }\end{array}$ & Hierarchical & Poisson & $\begin{array}{c}\text { Normal, } \\
\text { High }\end{array}$ & Yes & 2-D Disk & Yes & No & Yes & No & No & No & No \\
\hline ESS & Hierarchical & Any & $\begin{array}{c}\text { Normal, } \\
\text { High }\end{array}$ & Yes & Any & Any & No & Yes & No & No & No & No \\
\hline LDS & Hierarchical & Poisson & $\begin{array}{c}\text { Normal, } \\
\text { High }\end{array}$ & Yes & 2-D Disk & Yes & Yes & Yes & No & No & No & Yes \\
\hline BS & Hierarchical & Poisson & $\begin{array}{c}\text { Normal, } \\
\text { High }\end{array}$ & Yes & 2-D Disk & Yes & Yes & Yes & No & No & No & Yes \\
\hline
\end{tabular}

TABLE II

Classification BASEd ON OBJeCtives

\begin{tabular}{|c|c|c|c|c|c|}
\hline \multirow[b]{2}{*}{ Schemes } & \multicolumn{5}{|c|}{ Objectives } \\
\hline & $\begin{array}{l}\text { Sensing Coverage } \\
\text { Guarantee }\end{array}$ & $\begin{array}{c}\text { Network Connectivity } \\
\text { Guarantee }\end{array}$ & Data Delivery Ratio & Stealthiness & $\begin{array}{c}\text { Balanced Energy } \\
\text { Consumption }\end{array}$ \\
\hline $\begin{array}{c}\text { RIS } \\
\text { (Kumar) }\end{array}$ & Full, K, Asymptotic & & & Yes & Yes \\
\hline $\begin{array}{l}\text { Sponsored } \\
\text { Sector }\end{array}$ & Full, Original, Hard & & & Yes & Yes \\
\hline MSNL & Full, K, Hard & & & & Yes \\
\hline LDAS & Partial, 1, Statistical & & & & Yes \\
\hline PEAS & & 1, Hard & & & \\
\hline$\overline{\text { OGDC }}$ & Full, 1, Hard & 1, Hard & & Yes & Yes \\
\hline $\mathrm{CCP}$ & Full, K, Hard & K, Hard & & & Yes \\
\hline ASCENT & & & Yes & & \\
\hline PECAS & & 1, Hard & & & Yes \\
\hline LEACH & & & & $\overline{Y e s}$ & Yes \\
\hline E-LEACH & & & & Yes & Yes \\
\hline $\begin{array}{l}\text { Bandyo- } \\
\text { padhyay et } \\
\text { al. [2], [3] }\end{array}$ & & & & Yes & \\
\hline ESS & & & & Yes & Yes \\
\hline LDS & & & & & \\
\hline BS & & & & & Yes \\
\hline
\end{tabular}

\section{CONCLUSIONS}

We have reviewed 15 energy-efficient scheduling mechanisms that are designed specifically for sensor networks. We examined their design assumptions and objectives to find their commonalities and differences. We found that designers have to make many design assumptions about sensors' capabilities, sensor network structure and sensor deployment strategy, either explicitly or implicitly. Their design objectives also vary from simply maintaining sensing coverage to satisfying several
QoS objectives simultaneously. We believe that it is important to design a sensor network that can support a wide range of applications and such a network would have only one or a few generic scheduling mechanisms that can be tailored to different applications. In our future work, we plan to study the feasibility of this approach.

Furthermore, due to limited space of this conference paper, we have omitted many details of the surveyed papers, which will be included in the journal version [23] of this paper. 


\section{REFERENCES}

[1] I. Akyildiz, W. Su, Y. Sankarasubramaniam, and E. Cayirci. A survey on sensor networks. IEEE Communications Magazine, 40(8):102-114, Aug. 2002.

[2] S. Bandyopadhyay and E. J. Coyle. An energy efficient hierarchical clustering algorithm for wireless sensor networks. In INFOCOM, 2003

[3] S. Bandyopadhyay and E. J. Coyle. Minimizing communication costs in hierarchically-clustered networks of wireless sensors. Computer Networks, 44:1-16, 2004.

[4] P. Berman, G. Calinescu, C.Shah, and A. Zelikovsly. Efficient energy management in sensor networks. In Y. Xiao and Y. Pan, editors, Ad Hoc and Sensor Networks. Nova Science Publishers, 2005.

[5] N. Bulusu, J. Heidemann, and D. Estrin. GPS-less low cost outdoor localization for very small devices. IEEE Personal Communications Magazine, 7(5):28-34, October 2000.

[6] A. Cerpa and D. Estrin. Ascent: Adaptive self-configuring sensor networks topologies. In Proceedings of IEEE INFOCOM 2002, New York, NY, June 2002.

[7] Crossbow. Mpr/mib mote hardware users manual. http://www . xbow. com/Support/manuals.htm, aug 2004.

[8] Crossbow. Power management and batteries. http://www . xbow . com/Support/appnotes. htm, 2004.

[9] J. Deng, Y. S. Han, W. B. Heinzelman, and P. K. Varshney. Balancedenergy sleep scheduling scheme for high density cluster-based sensor networks. Elsevier Computer Communications Journal, Special Issue on ASWN '04, 2004. to appear.

[10] J. Deng, Y. S. Han, W. B. Heinzelman, and P. K. Varshney. Balancedenergy sleep scheduling scheme for high density cluster-based sensor networks. In Proc. of the 4th Workshop on Applications and Services in Wireless Networks (ASWN'04), 2004.

[11] J. Deng, Y. S. Han, W. B. Heinzelman, and P. K. Varshney. Scheduling sleeping nodes in high density cluster-based sensor networks ACM/Kluwer Mobile Networks and Applications (MONET) Special Issue on "Energy Constraints and Lifetime Performance in Wireless Sensor Networks", 2004. to appear.

[12] J. Elson and D. Estrin. Time synchronization for wireless sensor networks. In Proceedings of the 15th International Parallel \& Distributed Processing Symposium (IPDPS '01), 2001.

[13] C. Gui and P. Mohapatra. Power conservation and quality of surveillance in target tracking sensor networks. In Proceedings of the 10th Annual International Conference on Mobile Computing and Networking (Mobicom '04), pages 129-143, 2004.

[14] T. He, S. Krishnamurthy, J. A. Stankovic, T. Abdelzaher, L. Luo, R. Stoleru, T. Yan, L. Gu, J. Hui, and B. Krogh. Energy-efficient surveillance system using wireless sensor networks. In Proceedings of the 2nd International Conference on Mobile Systems, Applications, and Services (MobiSys '04), pages 270-283, 2004.

[15] W. Heinzelman, A. Chandrakasan, and H. Balakrishnan. An applicationspeciffic protocol architecture for wireless microsensor networks. IEEE Transactions on Wireless Communications, 1(4):660-670, Oct. 2002.

[16] W. R. Heinzelman, A. Chandrakasan, and H. Balakrishnan. Energyefficient communication protocols for wireless microsensor networks. In Proceedings of Hawaaian International Conference on Systems Science, Jan. 2000.

[17] J. Hill, R. Szewczyk, A. Woo, S. Hollar, D. Culler, and K. Pister. System architecture directions for networked sensors. In Proceedings of Ninth International Conference on Architectural Support for Porgramming Languages and Operating Systems, Nov. 2000.

[18] S. Kumar, T. H. Lai, and J. Balogh. On k-coverage in a mostly sleeping sensor network. In Proceedings of the 10th Annual International Conference on Mobile Computing and Networking (Mobicom '04), pages 144-158, 2004

[19] R. Powers. Batteries for low power electronics. Proceedings of the IEEE, pages 687-693, April 1995.

[20] E. Shih, S.-H. Cho, N. Ickes, R. Min, A. Sinha, A. Wang, and A. Chandrakasan. Physical layer driven protocol and algorithm design for energy-efficient wireless sensor networks. In Proceedings of ACM Mobicom '01, pages 272-287, 2001.

[21] S. Slijepcevic and M. Potkonjak. Power efficient organization of wireless sensor networks. In IEE ICC 2001, Helsinki, Finland, June 2001.

[22] D. Tian and N. D. Georganas. A coverage-preserving node scheduling scheme for large wireless sensor networks. In Proceedings of the 1st ACM International Workshop on Wireless Sensor Networks and Applications (WSNA '02), pages 32-41, 2002.

[23] L. Wang and Y. Xiao. A survey of energy saving mechanisms in sensor networks. ACM/Kluwer Mobile Networks and Applications (MONET) Special Issue on "Selected papers from Broadnets 2005”. submitted.

[24] X. Wang, G. Xing, Y. Zhang, C. Lu, R. Pless, and C. Gill. Integrated coverage and connectivity configuration in wireless sensor networks. In Proceedings of the 1st International Conference on Embedded Networked Sensor Systems (Sensys '03), pages 28-39, 2003.

[25] K. Wu, Y. Gao, F. Li, and Y. Xiao. Lightweight deployment-aware scheduling for wireless sensor networks. ACM/Kluwer Mobile Networks and Applications (MONET) Special Issue on "Energy Constraints and Lifetime Performance in Wireless Sensor Networks", 2004. to appear.

[26] G. Xing, C. Lu, R. Pless, and J. A. O'Sullivan. CoGrid: an efficient coverage maintenance protocol for distributed sensor networks. In Proceedings of IPSN 2004), Berkeley, CA, Apr. 2004

[27] F. Ye, G. Zhong, J. Cheng, S. Lu, and L. Zhang. Peas: A robust energy conserving protocol for long-lived sensor networks. In Proceedings of the 23rd International Conference on Distributed Computing Systems (ICDCS '03), pages 28-37, 2003.

[28] H. Zhang and J. C. Hou. Maintaining sensing coverage and connectivity in large sensor networks. In Proceedings of NSF International Workshop on Theoretical and Algorithmic Aspects of Sensor, Ad Hoc Wireless, and Peer-to-Peer Networks, 2004. invited paper. 